\title{
Self-organized Monolayer of Nanosized Ceria Colloids Stabilized by Poly(vinylpyrrolidone)
}

Rui Si, ${ }^{\dagger}$ Ya-Wen Zhang, ${ }^{* \dagger}$ Li-Ping You, ${ }^{\dagger}$ and Chun-Hua Yan*,†

† State Key Lab of Rare Earth Materials Chemistry and Applications \& PKU-HKU Joint Lab on Rare Earth Materials and Bioinorganic Chemistry, Peking University, Beijing 100871, China. E-mail: yan@pku.edu.cn.

* Electron Microscopy Laboratory, Peking University, Beijing 100871, China

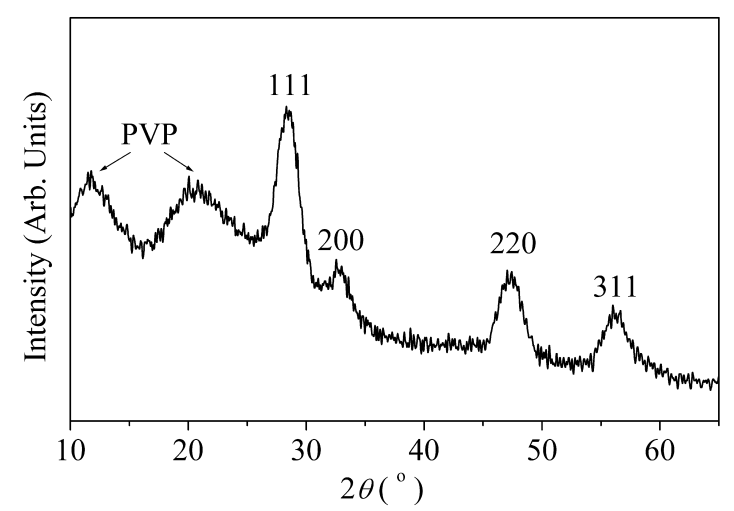

Figure 1S. XRD pattern of the colloids powder synthesized using triethylamine under $C_{\mathrm{PVP}}=30 \mathrm{~g} / \mathrm{L}$ at $180{ }^{\circ} \mathrm{C}$ for $24 \mathrm{~h}$ in ethanol, then precipitated by adding an excess amount of water-acetone mixed solvent $(\mathrm{v} / \mathrm{v}=1: 10)$ followed by drying at $80{ }^{\circ} \mathrm{C}$ in air overnight, from which, the formation of pure cubic $\mathrm{CeO}_{2}$ nanoparticles (JCPDS card No. 34-0394) and the presence of PVP in the colloidal product can be verified.

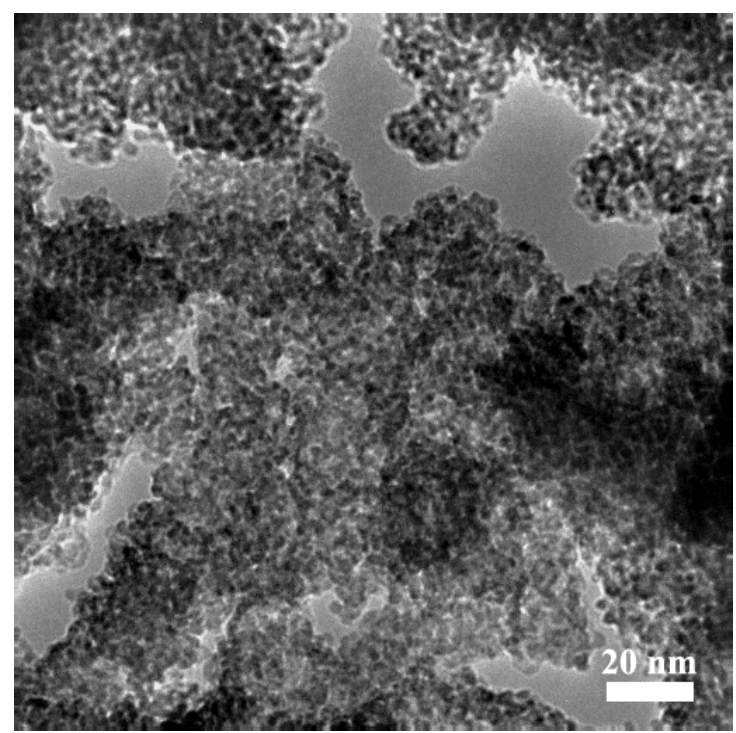

Figure 2S. TEM image of the precipitated brown $\mathrm{CeO}_{2}$ product synthesized from $\mathrm{Ce}\left(\mathrm{NO}_{3}\right)_{3}$ and $\mathrm{KOH}$, in the absence of PVP, indicating that the $\mathrm{CeO}_{2}$ nanoparticles are severely aggregated in random order. 


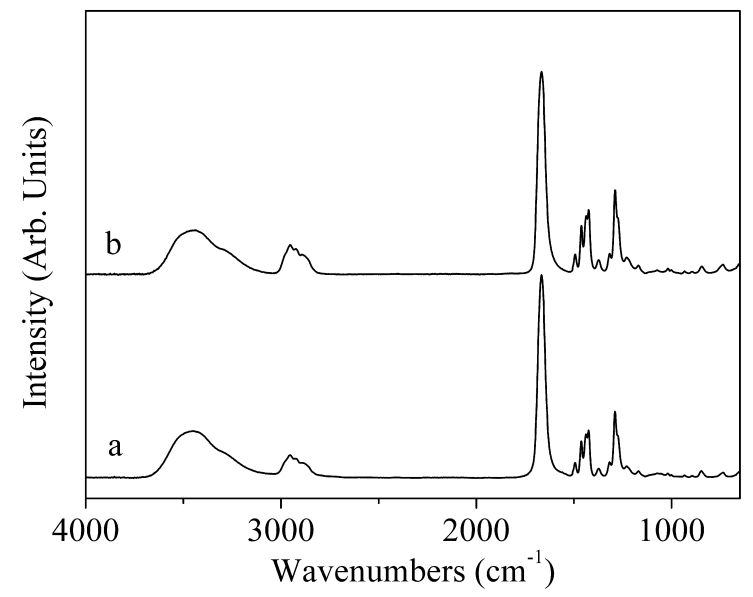

Figure 3S. IR spectra of the as-obtained ceria colloids (a) and PVP (b), indicating that PVP was predominately adsorbed onto the surface of the colloidal $\mathrm{CeO}_{2}$ nanoparticles. 